Review Article

\title{
GAGGING A REVIEW
}

\author{
Laxman Singh Kaira ${ }^{1}$, Esha Dabral ${ }^{2} \&$ Harpreet Singh Kukreja ${ }^{3}$ \\ ${ }^{1}$ Assistant Professor, Department of Dentistry, ${ }^{2}$ Consultant Dental Surgeon, Veer Chandra Singh Garhwali Government \\ Medical Sciences and Research Institute, Srinagar Garhwal, Uttrakhand, \\ ${ }^{3}$ Consultant Prosthodontist, Department of Prosthodontics, Udaipur, Rajasthan, INDIA. \\ Correspondence \\ Laxman Singh Kaira \\ Faculty Residence, Flat nu 4, Type 2, Veer Chandra Singh Garhwali Government M edical Sciences and Research Institute \\ Srinagar Garhwal, Uttrakhand. M obile : +918755902525 E-mail : laxmansingh6@gmail.com
}

\begin{abstract}
:
The gag reflex is a complex physiologic phenomenon. The problem compromises the quality of dental treatment and is a barrier to optimal patient care. The function of the reflex is protective in nature. When the reflex is abnormally active, the dentist may be presented with a bewildering and frustrating problem in various dental procedures, resulting in a strong potential for compromised treatment. The technique or techniques used should be dictated by the cause or causes involved. If organic disturbances, anatomic anomalies, or bio mechanical inadequacies of the existing prosthesis are not key causes the services of trained specialists are needed to help with behavioral management of the problem. A review of management of patients follows and includes strategies to assist clinicians.
\end{abstract}

Keywords: gagging, vomiting

\section{Introduction:}

Gagging has been defined as an ejectory constriction of the muscles of the pharyngeal sphincter. It is a normal protective reflex designed to protect the airway and remove irritant material from the posterior oropharynx and upper G.I.T. Gagging reaction range from mild choking to violent, uncontrolled retching which may/may not precede vomiting' Feintuch has described clinically the graphic description of a patient with gag reflex. "As the body trembles and footrest is stamped, large tears roll down from the eyes. The face of the victim takes on the hue of apoplectic purple and the patient gasps for breath, at the same time attempting to eject the introduce from his mouth and his insides with them". When spasmodic contraction of respiratory muscles occur during retching, air is forced through the closed glottis, producing the characteristic retching

Access this article online Quick Response Code

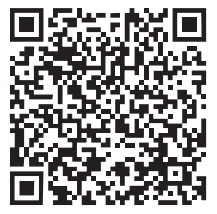

sound. In addition, chest muscles are in fixation and thoracic inlet muscles are contracting. This causes impediment of the venous return, dilating the veins of the head and neck, with flushing and congestion of the face. Active gag reflex upsets the patient, compromises quality of treatment and frustrates the dentist. Effective management of gagging depends on treatment of the cause and not merely symptoms. By thorough examination, taking of adequate medical history, and conversation with the patient, the dentist needs to determine if the patient's problem is related to iatrogenic factors, organic disturbances, anatomic or psychological factors. It is important to recognise whether, single or multiple factors are causing the problem. ${ }^{3}$

\section{Classification of Gagging}

Faigenblum ${ }^{5}$ classification of patients with gag reflex differentiates mild from severe retching. The patient with mild retching may experience nausea with minimal reaction to a stimulus and generally is able to control the response. Fortunately most patients fall in this category. Present from birth the gag reflex can be compared to the swallowing reflex. Swallowing occurs when the muscle action is smooth and co ordinate, when muscle action is smooth and unco ordinate gagging occurs.

M orstad gave a classification of gagging based on whether 
gagging occurs in a patient immediately after giving the prostheses or after a delayed period. Immediate - It is caused by overextension at post palatal area in maxillary denture or bulky distolingual flange in mandibular denture. It occurs when the prosthesis is given to the patient. Delayed - It occurs within two weeks to two months after insertion of the denture and may be due to an incomplete border seal which allows seepage of saliva under the denture.

\section{The Nature of Gagging}

The gag reflex is a normal healthy defence mechanism. It functions to prevent foreign bodies

from entering the trachea. Some clinicians suggest that not all regions of the mouth are equally sensitive to stimuli that produce the gag reflex. Yet5 regions of maximum sensitivity are identified as trigger areas. These are the fauces, base of the tongue, palate, uvula and posterior pharyngeal wall.

A clinical description of gagging behaviour has been described:

1. Puckering of the lips or attempting to close the jaws.

2. Elevating of furrowing the tongue, with rotation from back to front and with the hyoid bone at the centre.

3. Elevation of soft palate and hyoid bone.

4. Fixation of the hyoid bone.

5. Closing of the nasopharynx by an approximation of the posterior pillars of the fauces that elevate the soft palate.

6. Contraction of the anterior and posterior pillars of the fauces, causing the tonsils to rotate in an anteriomedial direction.

7. Elevation, contraction and retraction of the larynx and closure of the glottis.

8. Retching or simultaneous and uncoordinated respiratorymuscle spasm, and

9. Vomiting.

Other reflex behaviours that are extra oral in nature also can be observed by the clinician. These include excessive salivation, lacrimation, coughing and sweating. At times a full body response may occur. The patient extends the head, arms in an attempt to completely with draw from the attending stimuli, if the stimulus is repeated, collapse may occur for the extremely apprehensive or excited patient.

\section{Neural Involvement in Gagging:}

When stimulation occurs of the soft palate or posterior third of the tongue, afferent impulses are transmitted to a centre in the medulla oblongata. From this centre, efferent impulses arise and are transmitted, resulting in the spasmodic and uncoordinated movements of gagging. The centre in medulla oblongata is very close to the vomiting, salivating and cardiac centre, explaining why gagging may be accompanied by additional reflex activity (Ex.Drooling, tearing)

\section{Aetiology}

\section{Systemic Disorders:15, 16, 17}

_ Deviated septum, nasal polyps or sinusitis block nasal passages and increase likelihood of the gag reflex.

_ Alcoholism, smoking, chronic gastritis, carcinoma of the stomach, peptic ulcer, and cholecystics are related to chronic gastrointestinal irritability and gagging.

- Medication that a person may be taking are another consideration if this produce nausea as a side effect.

_ Diaphragmatic hernia has also been implicated as a systemic cause for gagging.

\section{Psychological Factors:}

Patients may gag to gain attention from the dentist, to avoid treatment, and/or to avoid the outcome of treatment. Further, fear is the underlying factor influencing the psychological gagger. The fear may be generalized and vague or quite specific. Often the fear is not merely that of pain. Some patients gag because of an abnormal fear of swallowing a foreign object. Study by WRIGHT on 53 retches provides no suggestions that people who retch during dental treatment or while wearing dentures are particularly neurotic. 


\section{Physiologic Factors:}

These can be read and understood if divided into extra oral and intraoral stimuli.

EXTRAORAL STIMULI: Visual, auditory and objector stimuli are extra oral factors that can elicit the gag reflex. The sight of a mouth mirror or impression tray is stimulus enough to cause some patients to gag. Landa ${ }^{4}$ observed a deaf patient suffer a spasm of gagging while observing the gagging of another patient. An acoustic stimulus can instigate a gag reflex in some patients. Landa describes a husband and wife who were both severe gaggers. The sound of the wife gagging was sufficient to precipitate an attack of gagging in the husband, who was seated in another operatory. Certain smells may cause a patient to gag. The smell of various dental substances, cigarette smoke etc have been reported to cause gag reflex.

INTRAORALSTIM ULI: The effect of tactile stimuli gag reflex is well known. Patients show considerable variation in the ability to withstand various tactile stimuli. The palate is roughly divided into two response regions for tactile irritation hyposensitive and hypersensitive. A line drawn through the fovea palatine demarcates the relatively hyposensitive anterior portion from the hypersensitive posterior portion. The tongue may be similarly divided into a hypersensitive anterior region and a hypersensitive posterior one third. It has been stated that the upper surface of the posterior $1 / 3$ of the tongue is the most sensitive region in the entire oral cavity.

\section{4. latrogenic Stimuli:}

Tactile stimulation of the oral tissues inevitably occurs when executing various dental procedures, careful examination and execution of modified techniques can minimize stimulation that causes gagging. However, gagging difficulties caused by crowns or fixed partial dentition are not reported in the dental literature. Because of their more obstructive nature, complete dentures have drawn all the attention. Biomechanical aspects related to gagging are inadequate post dam, over extended posterior borders, inharmonious occlusion, poor retention, surface finish of acrylic and inadequate free way space.
Inadequate Post Dam: Produces gagging as a result of too little pressure applied to the palatal tissue. When a post dam is to shallow, the tight pressure that results, can produce a tickling sensation which elicits a gag.

Over extended Borders: The posterior part of the maxillary denture and the distolingual part

of the mandibular denture may impinge on, one, or more trigger areas and cause gagging.

Occlusion: Landa ${ }^{4}$ suggests that correct occlusion and balanced articulation are critical for minimizing the gag reflex. A stable occlusion causes the denture to compress the underlying tissues. Conversely, instable occlusion produces movement of the denture base, which in this produce atickling sensation and gagging.

\section{Causes}

Several authors propose a number of general classifications of causes which include extra oral and intraoral, physiologic and iatrogenic factors.

\section{Systemic Disorder:}

The patients general health is often related to dental health with some impact on the gagging reflex Chronic conditions, such as deviated septum, nasal polyps, or sinusitis, blocked nasal passages increase the likelihood of the gag reflex. Chronic problems of gastrointestinal tract may increase irritability, lower the threshold for excitation of the oral cavity and contribute to nausea and gagging. Alcoholism, Chronic gastritis and carcinoma of stomach, peptic ulcer and cholecystitis are related to chronic gastro intestinal irritability and gagging. Inflammation of the pharynx is a hypersensitive gag reflex. This condition is common in persons who drink and smoke excessively. Medications that the patient may be taking are another consideration if they produce nausea as a side effect.

\section{Psychologic Factors:}

Discussing the psychologic aspects of gagging, Bartlett ${ }^{2}$ states that such psychosomatic reaction may be active or passive. An active reaction is due to factors that currently have some functional purpose in the patient's life situation. 
For various psychologic reasons, patients may gag to gain attention from the dentist, to avoid treatment, and or to avoid the outcome of treatment. In contrast, a passive reaction is the result for various reasons, the causes of which are no longer functionally important. Wright ${ }^{2}$ concluded that one third of the patients reported the problem as being most acute in the morning during oral hygiene and insertion of dentures. This might occur from lack of habituation to stimulation from the denture since it was not worn at night. Kramer and Braham stated that "fear" is almost always the underlying factor influencing the psychologic gagger.

\section{Physiologic Factors:}

Visual, auditory and factory stimuli are extraoral factors that can elicit the gag reflex, while dental prostheses and performance of dental procedures represent intraoral stimuli

(a) Extraoral Stimuli : The mere sight of a mouth mirror or impression tray is stimulus enough to cause some patients to gag. Landa observed a deaf patient suffer a spasm of gagging while viewing gagging of another patient.

(b) Intraoral Stimuli : Landa states that posterior part of palate and upper surface of the posterior one third of the tongue are the most sensitive regions in the entire oral cavity. Tactile stimulation of the oral tissues inevitably occurs when executing various dental procedures. In the dental literature, various biomechanical aspects of dental prosthesis are suggested as causing the gag reflex. These biomechanical aspect related to gagging are inadequate post dam, over extended posterior borders, disharmonious occlusion, poor retention, surface finish of acrylic resin and inadequate free way space. An under extended prosthesis also may contribute to a gagging problem. Inadequately extended borders that result in poor retention produce an unstable prosthesis. Movement of such prostheses may stimulate tickling sensation and elicit a gag. Krol ${ }^{1}$ stated that inadequate free way space may cause gagging related to complete dentures and his explanation suggested that the elevator muscles do not relax normally if the occlusal vertical dimension exceeds the rest vertical dimension.
This may cause a spasm that sets in action a chain of swallowing muscle responses. One possible sequence would involve a spasm of the tensor palatini muscles, which produces sensation when the maxillary denture seems to extend too far backward. The tensor palatini muscles slightly depresses the soft palate and presses it against the posterior border of the denture, producing gag reflex.

\section{IV latrogenic Factors:}

In the otherwise non gagging patient, poor execution of intra oral procedures may elicit gag reflex. Sensitive tissues may be stimulated as a result of rough or careless technique and temperature extremes of instruments.

\section{M anagement:}

Effective management of gagging depends over treating the cause and not merely the symptoms treatments falls into following categories, namely (1) Psychologic intervention (2) Prosthodontic management (3) Pharmacologic measures (4) Surgical intervention (5) Accupressure and Accupunture.

\section{(1) Psychologic Intervention}

The spectrum of suggested approaches runs from a gentle manner to psychotherapy. Psychotherapy includes hypnosis, behavior management procedure of systemic desensitizing, covert reinforcement modelling fear reduction. Many recommended clinical techniques are directed at diverting the patients attention from the gagging stimuli. Landa ${ }^{4}$ suggested that the

dentist engage the patient in conversation on some to pic of special interest. Kovats ${ }^{9}$ reported a technique that has the patient breathe audibly through the nose and at the same time, rhythmically tap the right foot on the floor. By concentrating on these activities the patient's attention may be diverted away from the gagging stimulus. Faigenblum ${ }^{9}$ stated that vomiting is impossible during apnea. To control gagging the patient should be instructed to prolong the expiratory effort at the expense of inspiration. This will produce a state of apnea and discourage gagging. A similar technique was described by 
$\mathrm{Krol}^{1}$ to divert attention, the patient is instructed to raise his or her leg and hold it in the air. As the patient's muscles become increasingly fatigued, more and more conscious effort is required to hold the leg up. At the point where the patient has difficulty carrying on conversation, intraoral procedures may be attempted.

\section{Prosthodontic M anagement:}

Prosthodontic approach to the patient with the gagging problem involve technical modifications to render the prosthesis more acceptable to the patient. Excess thickness, overextension or inadequate postdam should be corrected before more radical modifications in the prosthesis are made.

The smooth, shiny surface of a complete denture is objectionable to some patients. From his clinical experience, Jordan ${ }^{9}$ suggests that a matte finish is more acceptable to patients than a glossy surface. $\mathrm{Krol}^{1}$ discussed the importance of "Freeway" space to the gag reflex. In all instances, an increase in the interocclusal distance resolved the gagging problem. To avoid substandard impressions because of gagging, Brokin5 outlined an impression technique for edentulous patients. A primary impression is obtained by pouring Kerr impression wax. The pliable nature of the wax allows reseating of the tray and border molding until desirable results are obtained. A technique that employs ordinary marbles was reported by Singer ${ }^{7}$ as an effective approach to overcoming a patient's inability to tolerate complete dentures.

At the first appointment, the patient was asked to place five marbles in his mouth one at a time and at his leisure. The patient was further instructed to keep the marbles in his mouth continuously for 1 week, except when eating and sleeping. After 1 week patient's ability to tolerate the marbles was evaluated, and he was reassured that he would be able to tolerate denture. At the third visit modeling compound impression were made, refined and completed. At the fourth visit, the lower base tray was inserted along with three marbles in the mouth, and a "training bead" was placed on the lingual aspect of the base tray to maintain proper tongue position. During the fifth visit the use of marbles was discontinued, and at the sixth visit, jaw records were made and the occlusion rims marked. The completed dentures were inserted at the seventh visit. Singer admits that patient's motivation is an indispensable component of the marble technique. It appears that his approach presents definite medicolegal risks in the event of aspiration by the patient.

\section{Training Bases}

This is a desensitization technique, whereby a patient is progressively supplied with a series of small to full-sized denture bases. A thin acrylic denture base, without teeth, is fabricated and the patient is asked to wear it at home, gradually increasing the length of time the training base is worn. A suitable regime maybe 5 minutes once each day, then twice each day and so on. After 1 week the patient is asked to increase this to 10 minutes 3 times each day, then 15 minutes, 30 minutes, and 1 hour. Eventually the patient is able to tolerate the training base for most of the day. The timing and rate of progress will vary between patients, depending upon individual needs and expectations. Anterior teeth are added to the original or an extended training base and, when the patient is able to tolerate this, posterior teeth are added. Palateless dentures have been shown to be effective in some patients and loss of retention is not always significantly affected. Some authors, however would still only recommend this option as a last resort.

\section{Cognitive Behavioral Therapy}

This method focuses on changing irrational thought processes. For example, some patients retch when water from the highspeed hand piece is felt. When questioned, it is not unusual for an individual to admit to a fear of choking, believing that breathing will stop, resulting in death. Some patients may believe that the fear of dentistry will cause a fatal heart attack. Acognitive behavioral psychotherapist willbattempt to rationalize these thought patterns in patients with persistent psychogenic gagging.

\section{Pharmacologic Measures:}

The drugs used to control gagging maybe classified as peripherally acting or centrally acting drugs. Peripherally acting drugs are topical and local anesthetics. The rationale 
for the use of these drugs is that if the afferent impulses from sensitive oral tissues are eliminated the reflex of gagging will not take place. Centrally acting drugs categorized as antihistamines, sedatives and tranquilizers, parasympatholytics, and central nervous system depressants. Pharmacologic intervention offers only a short-term solution, aspecially for severe, chronic problems.

\section{Conscious Sedation}

When a disruptive gag reflex is thought to be a manifestation of anxiety, removal of the anxiety may prevent gagging. The use of conscious sedation with inhalation, oral, or intravenous agents may temporarily eliminate gagging during dental treatment while maintaining reflexes that protect the patient's airway. However, while sedation may allow adequate treatment to be performed, it will not help the patient overcome retching if, for example, a prosthesis must be worn. The use of oral sedatives may be unpredictable and is usually only useful in the mild gagging patient with an underlying anxiety state. Intravenous sedation is often much more predictable than oral sedation, and can be of use in patients where inhalation sedation is ineffective.

\section{Surgical Corrections:}

Leslie $^{10}$ reported a surgical technique to relieve gagging for the patient unable to tolerate complete dentures. The basis for this technique stems from the observation that persistent gagging results from an atonic and relaxed soft palate, which is found in the nervous patient. To correct this situation, Leslie advocated an operation to shorten and tighten the soft palate.

\section{The Role of Accupuncture :}

Acupuncture is a system of medicine in which a fine needle is inserted through the skin to a depth of a few millimetres, left in place for a time, sometimes manipulated and then withdrawn. Acupuncture is one of a range of treatment options that can be employed in an effort to control gagging. There is a specific, recognized anti-gagging point on the ear. The technique involves the insertion of one, fine, single-use disposable needle of $7 \mathrm{~mm}$ length into the anti-gagging point of each ear to a depth of $3 \mathrm{~mm}$. The needles are manipulated for 30 seconds prior to carrying out dental treatment. The needles remain in situ throughout the treatment and are removed before the patient is discharged. The patient does not require an escort. The technique is said to be safe, quick, inexpensive and relatively non-invasive.

\section{Accupressure Techniques:}

Accupressure technique has been described as effective in controlling gag reflexduring dental procedures. Chengjiang (REN-24) is an effective acupressure point for controlling the gag reflex. REN-24 point is located in the horizontal mentolabial groove, approximately midway between the chin and the lower lip. Light finger pressure is applied with the index finger. Progressively the finger pressure is increased until the patient feels discomfort.

The accupressure procedures should start at least 5 minutes before impression making, continue through the impression procedure and be terminated only after the impression has been removed from the patient's mouth. Pressure can be applied by the patient, dental assistant or dentist. Accupressure caves are sensitive points in the human body that feel soreness distention, when deep pressure is applied for five to twenty minutes. These points are left and right concave area at medial aspect of the forearm and concave area between first and second metacarpal bones.

\section{Summary and Conclusion:}

The most serious problem associated with the strong potential with an overactive gag reflex is the strong potential for compromised treatment and it presents as a challenge to the capability of a dentist. M any techniques are available for controlling the exaggerated gag reflex and no single technique will solve each patient's problem. The conscious mind of the patient must be regarded by the dentist as the primary factors for the inhibition of gagging. Building a relation based on confidence is more valuable than applying most medicaments. 


\section{References:}

1. Krol, A. J. : A new approach to the gagging problem J. Prosthet Dent 13: $611,1963$.

2. Daniel J. Conny and Lisa - A. Tedesco: The gagging problem in prosthodontic treatment Part I : Description and causes J. Prosthet Dent; 49(5): 601, 1983.

3. R. D. Savage and A. R. M acgregor : Behavior Therapy in Prosthodontics J. Prosthet Dent; 24(2): 126-131,1970.

4. LandaJ. S. : Practical full Denture Prosthesis: Lond, 1954.

5. Borkin. D. N. : Impression technique for patients that gag: J. Prosthet Dent 9:386,1959.

6. Schole M . L. : M anagement of the gagging patient J. Prosthet Dent 9: 5, 1959.

7. Singer I. L. : The Marble Technique: A method for treating the "hopeless gager for complete dentures. J. Prosthet Dent $29: 146$, 1973.

8. Craig R. M eans, Ingeborg E. Flenniken. Gagging a problem in prosthetic DentistryJ. Prosthet Dent; $23(6): 614-619,1970$.

9. Daniel J. Conny and Lisa - A. Tedesco: The gagging problem in prosthodontic treatment Part II : Patient Management J. Prosthet Dent; 49(6): 757-761, 1983.

10. J. Fiske and C. Dickinson: The role of acupuncture in controlling the gagging reflex using a review of ten cases British Dent.J ournal; 01190 (11): 611-613, 2001. 